\title{
Nanoscale Structural Manipulation of Ion Irradiated Pyrochlore
}

\author{
J. Lian, L. M. Wang, and R. C. Ewing
}

Dept. of Nuclear Engineering \& Radiological Sciences, University of Michigan, Ann Arbor, MI 48109-2104

Ionic conductors are the basis of technologies that utilize solid-state electrochemical cells as a source of clean and efficient energy, such as in high-temperature batteries and fuel cells, and as sensors. $\mathrm{Gd}_{2}\left(\mathrm{Zr}_{\mathrm{x}} \mathrm{Ti}_{1-\mathrm{x}}\right)_{2} \mathrm{O}_{7}$ pyrochlore is an extrinsic ionic conductor at low values of $\mathrm{x}$, but it becomes an intrinsic fast-ion conductor at large $\mathrm{x}$, and oxygen vacancies are the dominant migrating species. Variations in the ionic and electronic conductivity of pyrochlore compounds, $\mathrm{A}_{2} \mathrm{~B}_{2} \mathrm{O}_{7}$, are related to disordering of the A- and B-site cations and the oxygen anion vacancies. A goal has been to increase ionic conductivity by increasing either the atomic-scale structural disorder or by fabricating nanoscale geometries that increase the boundary domain. Here, we intend to modify the electronic properties of pyrochlores by controlling the microstructure using an ion beam irradiation-induced order-disorder transformation in pyrochlore.

Fig. 1A shows a cross-sectional TEM image of $\mathrm{Gd}_{2} \mathrm{Ti}_{2} \mathrm{O}_{7}$ single crystal irradiated by $1.5 \mathrm{MeV} \mathrm{Xe}{ }^{+}$at a dose of $8.6 \times 10^{13}$ ions $/ \mathrm{cm}^{2}$. A completely amorphous layer was produced near the surface, and its depth $(\sim 300 \mathrm{~nm})$ is in good agreement with the range calculated using TRIM-96. Below the amorphous layer, however, there is a mixed domain of pyrochlore and fluorite structure-types, as indicated by the SAED patterns. The pyrochlore and fluorite structures can be distinguished by the presence or absence of superlattice diffraction maxima (weak diffraction spots in SAED). This disordered fluorite is created by A- and B-site cation disordering induced by ion beam irradiation. By varying the energy and mass of implanted ion species, we are able to precisely control the depth and width of damage layer in rare-earth titanate pyrochlores. An amorphous layer with slightly smaller thickness $(\sim 200 \mathrm{~nm})$ than that of $\mathrm{GdTi}_{2} \mathrm{O}_{7}$ formed in $\mathrm{Tm}_{2} \mathrm{Ti}_{2} \mathrm{O}_{7}$, as shown in Fig. 1B. For $\mathrm{Er}_{2} \mathrm{Ti}_{2} \mathrm{O}_{7}$ irradiated by $1 \mathrm{MeV} \mathrm{Kr}{ }^{+}$at a dose of $1.74 \times 10^{14}$ ions $/ \mathrm{cm}^{2}$, a highly damaged layer was created (Fig. 2A), which consists of nano-size disordered fluorite, as evidenced by the HRTEM image (Fig. 2B). Furthermore, using a $1.5 \mathrm{MeV} \mathrm{Xe}$ irradiation of a single crystal of $\mathrm{Gd}_{2} \mathrm{Ti}_{2} \mathrm{O}_{7}$ at a dose of $8.6 \times 10^{12}$ ions $/ \mathrm{cm}^{2}$, which is less than the critical amorphization dose, we successfully produce a buried disordered fluorite layer with a thickness of $\sim 12 \mathrm{~nm}$ within ordered pyrochlore matrix. This has never been achieved by other techniques. In contrast to the pyrochlore substrate, the lattice constant of the buried fluorite-structure layer is one-half that of the pyrochlore substrate. This buried disordered fluorite layer has a coherent interface with the ordered pyrochlore substrate, as confirmed by computer simulations shown in Fig. 3B (ordered pyrochlore) and Fig. 3C (defect fluorite).

These results demonstrate that by using ion beam techniques, we are able to manipulate the microstructure of pyrochlore compounds from amorphous layer and nano-size defect fluorite to a coherent nano-scale buried fluorite layer. This combines atomic-scale disorder on the cation and anion sites with a nanostructure that increases the interface area. This opens the possibility of developing new types of nano-scale mixed ionic-electronic conductors in pyrochlore ceramics and, specifically, in devices such as monolithic fuel cell structures. 

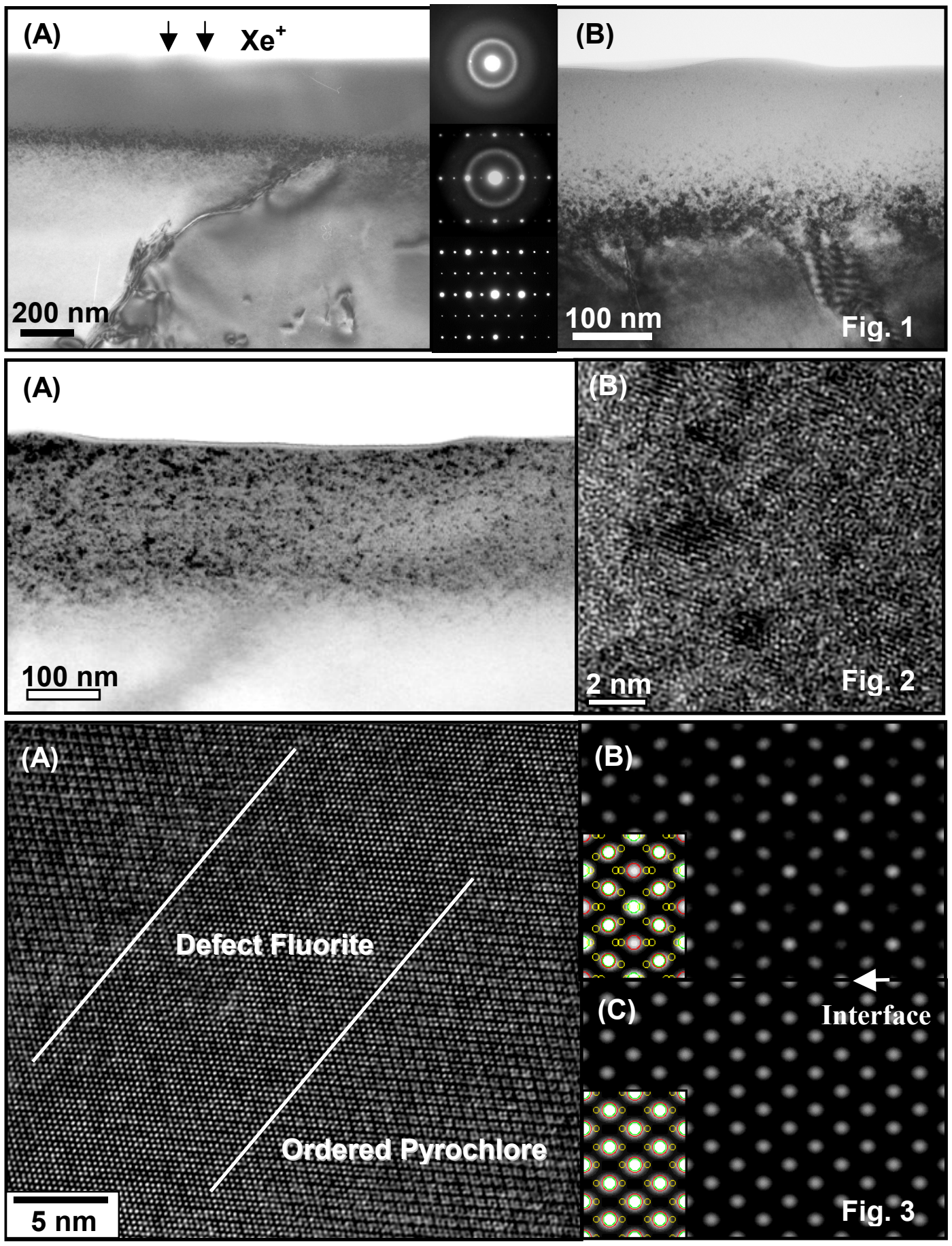

Fig. 1. Cross-section TEM images of $\mathrm{Gd}_{2} \mathrm{Ti}_{2} \mathrm{O}_{7}(\mathrm{~A})$ and $\mathrm{Tm}_{2} \mathrm{Ti}_{2} \mathrm{O}_{7}(\mathrm{~B})$ showing an amorphous layer under irradiation of $1.5 \mathrm{MeV} \mathrm{Xe}^{+}$ions at a dose of $8.6 \times 10^{13}$ ions $/ \mathrm{cm}^{2}$. SAED patterns inset are taken across the damage profiles in $\mathrm{Gd}_{2} \mathrm{Ti}_{2} \mathrm{O}_{7}$. Fig. 2. Cross-section and HRTEM images showing nano-size defect fluorite in $1 \mathrm{MeV} \mathrm{Kr}^{+}$irradiated $\mathrm{Er}_{2} \mathrm{Ti}_{2} \mathrm{O}_{7}$ at a dose of $1.74 \times 10^{14} \mathrm{ions} / \mathrm{cm}^{2}$.

Fig. 3. HRTEM image (A) showing a $\sim 12 \mathrm{~nm}$ defect fluorite buried layer in $\mathrm{Gd}_{2} \mathrm{Ti}_{2} \mathrm{O}_{7}$ irradiated by $1.5 \mathrm{MeV} \mathrm{Xe}{ }^{+}$at a doe of $8.6 \times 10^{12}$ ions $/ \mathrm{cm}^{2}$. HRTEM simulations with MacTempas showing a coherent interface between ordered pyrochlore (B) and defect fluorite (C), for which the insets are structural models for computer simulations. 